PROCEEDINGS OF THE

AMERICAN MATHEMATICAL SOCIETY

Volume 134, Number 1, Pages 117-127

S 0002-9939(05)08340-1

Article electronically published on August 22, 2005

\title{
A CONSTRUCTION OF A ROBUST FAMILY OF EXPONENTIAL ATTRACTORS
}

\author{
STEFANIA GATTI, MAURIZIO GRASSELLI, ALAIN MIRANVILLE, \\ AND VITTORINO PATA
}

(Communicated by David S. Tartakoff)

\begin{abstract}
Given a dissipative strongly continuous semigroup depending on some parameters, we construct a family of exponential attractors which is robust, in the sense of the symmetric Hausdorff distance, with respect to (even singular) perturbations.
\end{abstract}

\section{INTRODUCTION}

Exponential attractors for strongly continuous semigroups were first introduced in 2. The motivation comes from the lack of effective information given by global attractors, which present two major drawbacks, since they do not provide an actual control of the convergence rate of trajectories, and, consequently, they might be quite unstable with respect to perturbations (cf. [1]). The original technique to build exponential attractors was developed in Hilbert spaces, and it heavily relied on the use of orthogonal projections. Later, in [1, 3. new methods were introduced, that work in a Banach space setting.

A further interesting issue is to consider not just a single semigroup, but rather a family of semigroups depending on certain parameters. It is then of some importance to have results establishing good stability properties of the related exponential attractors, in dependence of the parameters. Significant achievements in this direction have been obtained in [4, 10, and, especially in [6], where the case of a singular perturbation has been successfully treated.

In this paper, we provide a stability result of the same kind, which takes into account the case of singular perturbations. What mostly motivated our investigation is that, in order to apply the methods of [6] to concrete problems arising from PDE, one has to construct some Banach spaces that are not natural, in the sense that they are not the spaces suggested by the equations. Besides, it might be quite difficult to understand which is the correct construction that works in a given setting. On the contrary, our technique provides a sort of "machinery" that can be automatically applied, using the correct spaces where the solutions live. As a

Received by the editors December 14, 2003

2000 Mathematics Subject Classification. Primary 37L25, 37L30.

Key words and phrases. Strongly continuous semigroups, robust exponential attractors, fractal dimension.

This research was partially supported by the Italian MIUR FIRB Research Project Analisi di Equazioni a Derivate Parziali, Lineari e Non Lineari: Aspetti Metodologici, Modellistica, Applicazioni. 
byproduct, we can obtain the desired results with much less computations than the ones required following the approach devised in [6]. In particular, we drastically simplify the analysis of the boundary layer 9 , which serves to control the difference between the perturbed and the limiting semigroups (see [6, 7]). This is indeed a nontrivial improvement, since the estimate of the boundary layer introduces relevant technical difficulties (of course, the study of the boundary layer has an interest of its own).

Before getting into details, some preliminary notations and definitions are in order. Let $S(t)$ be a strongly continuous semigroup acting on a Banach space $\mathcal{H}$. Recall that, for $\mathcal{B}_{1}, \mathcal{B}_{2} \subset \mathcal{H}$, the Hausdorff semi-distance between $\mathcal{B}_{1}$ and $\mathcal{B}_{2}$ is given by

$$
\operatorname{dist}_{\mathcal{H}}\left(\mathcal{B}_{1}, \mathcal{B}_{2}\right)=\sup _{b_{1} \in \mathcal{B}_{1}} \inf _{b_{2} \in \mathcal{B}_{2}}\left\|b_{1}-b_{2}\right\|_{\mathcal{H}}
$$

whereas the symmetric Hausdorff distance between $\mathcal{B}_{1}$ and $\mathcal{B}_{2}$ is

$$
\operatorname{dist}_{\mathcal{H}}^{\mathrm{sym}}\left(\mathcal{B}_{1}, \mathcal{B}_{2}\right)=\max \left\{\operatorname{dist}_{\mathcal{H}}\left(\mathcal{B}_{1}, \mathcal{B}_{2}\right), \operatorname{dist}_{\mathcal{H}}\left(\mathcal{B}_{2}, \mathcal{B}_{1}\right)\right\} .
$$

Given $r>0$ and a (relatively) compact set $\mathcal{K} \subset \mathcal{H}$, let us denote by $\mathcal{N}_{r}(\mathcal{K}, \mathcal{H})$ the minimum number of $r$-balls of $\mathcal{H}$ necessary to cover $\mathcal{K}$. Then, the fractal dimension of $\mathcal{K}$ is defined as

$$
\operatorname{dim}_{\mathcal{H}}[\mathcal{K}]=\limsup _{r \rightarrow 0} \frac{\ln \mathcal{N}_{r}(\mathcal{K}, \mathcal{H})}{\ln \frac{1}{r}} .
$$

Definition. Let $\mathcal{B} \subset \mathcal{H}$ be a bounded closed set, and let $t_{0} \geq 0$ be such that $S(t) \mathcal{B} \subset \mathcal{B}$, for all $t \geq t_{0}$. A compact set $\mathcal{M} \subset \mathcal{B}$ is called an exponential attractor or inertial set for $S(t)$ on $\mathcal{B}$ (with respect to the topology of $\mathcal{H}$ ) if the following conditions hold:

(i) $\mathcal{M}$ is (positively) invariant for $S(t)$, that is, $S(t) \mathcal{M} \subset \mathcal{M}$ for every $t \geq 0$.

(ii) $\operatorname{dim}_{\mathcal{H}}[\mathcal{M}]<\infty$.

(iii) There exist $M \geq 0$ and $\omega>0$ such that

$$
\operatorname{dist}_{\mathcal{H}}(S(t) \mathcal{B}, \mathcal{M}) \leq M e^{-\omega t}, \quad \forall t \geq 0 .
$$

Observe that $\mathcal{B}$ may not be necessarily invariant for $S(t)$.

\section{The TheOrem}

Notation. Let $X^{0}, X^{1}, X^{2}, Y^{0}, Y^{1}, Y^{2}$ be Banach spaces with embeddings

$$
X^{2} \subset X^{1} \Subset X^{0} \quad \text { and } \quad Y^{2} \subset Y^{1} \Subset Y^{0} .
$$

For every $\varepsilon \in\left[0, \varepsilon_{0}\right]$ and $j=0,1,2$, introduce the Banach spaces

$$
\mathcal{H}_{\varepsilon}^{j}=X^{j} \times Y^{j}
$$

endowed with the norms

$$
\|(x, y)\|_{\mathcal{H}_{\varepsilon}^{j}}^{2}=\|x\|_{X^{j}}^{2}+\varepsilon\|y\|_{Y^{j}}^{2} .
$$

It is understood that when $\varepsilon=0$ the above spaces reduce to the first component only. We denote by $\mathbb{B}_{\varepsilon}^{j}(z, r)$ the closed balls in $\mathcal{H}_{\varepsilon}^{j}$ of radius $r>0$ centered at $z$. Finally, let $\delta_{0} \geq 0$ be fixed. For every $\delta \in\left[0, \delta_{0}\right]$, let

$$
S_{\varepsilon, \delta}(t): \mathcal{H}_{\varepsilon}^{0} \rightarrow \mathcal{H}_{\varepsilon}^{0}
$$

be a strongly continuous semigroup of operators. 
In the sequel, $\mathbb{N}^{+}$and $\mathbb{N}$ will be the sets of positive and nonnegative integers, respectively.

The result is the following.

Theorem. Assume that there exist $\Lambda_{j} \geq 0, \alpha, \beta, \gamma, \vartheta \in(0,1], \kappa \in\left(0, \frac{1}{2}\right)$ and $\rho>0$ (all independent of $\varepsilon$ and $\delta$ ) such that, setting $\mathcal{B}_{\varepsilon}=\mathbb{B}_{\varepsilon}^{2}(0, \rho)$, the following conditions hold:

(H1) There exists a map $\mathcal{J}_{\delta}: \mathcal{B}_{0} \rightarrow Y^{0}$ Hölder continuous of exponent $\alpha$, uniformly with respect to $\delta$. Here, $\mathcal{B}_{0}$ is endowed with the metric topology of $\mathcal{H}_{0}^{0}$.

(H2) There exists $t^{*}>0$, independent of $\varepsilon$ and $\delta$, such that

$$
S_{\varepsilon, \delta}(t) \mathcal{B}_{\varepsilon} \subset \mathcal{B}_{\varepsilon}, \quad \forall t \geq t^{*} .
$$

Moreover, setting $S_{\varepsilon, \delta}\left(t^{*}\right)=S_{\varepsilon, \delta}$, the map $S_{\varepsilon, \delta}$ satisfies, for every $z_{1}, z_{2} \in$ $\mathcal{B}_{\varepsilon}$,

$$
S_{\varepsilon, \delta} z_{1}-S_{\varepsilon, \delta} z_{2}=L_{\varepsilon, \delta}\left(z_{1}, z_{2}\right)+K_{\varepsilon, \delta}\left(z_{1}, z_{2}\right)
$$

where

$$
\begin{aligned}
\left\|L_{\varepsilon, \delta}\left(z_{1}, z_{2}\right)\right\|_{\mathcal{H}_{\varepsilon}^{0}} \leq \kappa\left\|z_{1}-z_{2}\right\|_{\mathcal{H}_{\varepsilon}^{0}} \\
\left\|K_{\varepsilon, \delta}\left(z_{1}, z_{2}\right)\right\|_{\mathcal{H}_{\varepsilon}^{1}} \leq \Lambda_{1}\left\|z_{1}-z_{2}\right\|_{\mathcal{H}_{\varepsilon}^{0}} .
\end{aligned}
$$

(H3) The "lifting" map $\mathcal{L}_{\varepsilon, \delta}: \mathcal{B}_{0} \rightarrow \mathcal{H}_{\varepsilon}^{0}$, defined as

$$
\mathcal{L}_{\varepsilon, \delta} x= \begin{cases}\left(x, \mathcal{J}_{\delta} x\right), & \text { if } \varepsilon>0, \\ x, & \text { if } \varepsilon=0,\end{cases}
$$

fulfills

$$
\left\|S_{\varepsilon, \delta}^{n} z-\mathcal{L}_{\varepsilon, \delta} S_{0,0}^{n} \Pi_{\varepsilon} z\right\|_{\mathcal{H}_{\varepsilon}^{0}} \leq \Lambda_{2}^{n}(\varepsilon+\delta)^{\beta}, \quad \forall z \in \mathcal{B}_{\varepsilon}, \forall n \in \mathbb{N}^{+},
$$

where $\Pi_{\varepsilon}: \mathcal{B}_{\varepsilon} \rightarrow \mathcal{B}_{0}$ is the projection onto the first component when $\varepsilon>0$, and the identity map otherwise.

(H4) For any $z \in \mathcal{B}_{\varepsilon}$ there holds

$$
\left\|S_{\varepsilon, \delta}(t) z-\mathcal{L}_{\varepsilon, \delta} S_{0,0}(t) \Pi_{\varepsilon} z\right\|_{\mathcal{H}_{\varepsilon}^{0}} \leq \Lambda_{3}(\varepsilon+\delta)^{\gamma},
$$

for all $t \in\left[t^{*}, 2 t^{*}\right]$.

(H5) The map

$$
z \mapsto S_{\varepsilon, \delta}(t) z: \mathcal{B}_{\varepsilon} \rightarrow \mathcal{B}_{\varepsilon}
$$

is Lipschitz continuous on $\mathcal{B}_{\varepsilon}$, with a Lipschitz constant independent of $\varepsilon, \delta$ and $t \in\left[t^{*}, 2 t^{*}\right]$. Here, $\mathcal{B}_{\varepsilon}$ is endowed with the metric topology of $\mathcal{H}_{\varepsilon}^{0}$.

(H6) The map

$$
(t, z) \mapsto S_{\varepsilon, \delta}(t) z:\left[t^{*}, 2 t^{*}\right] \times \mathcal{B}_{\varepsilon} \rightarrow \mathcal{B}_{\varepsilon}
$$

is Hölder continuous of exponent $\vartheta$ (we do not require uniformity with respect to $\varepsilon$ and $\delta)$. Again, $\mathcal{B}_{\varepsilon}$ is endowed with the metric topology of $\mathcal{H}_{\varepsilon}^{0}$.

Then there exists a family of exponential attractors $\mathcal{M}_{\varepsilon, \delta}$ on $\widetilde{\mathcal{B}}_{\varepsilon}=\overline{\mathcal{B}}_{\varepsilon} \mathcal{H}_{\varepsilon}^{0}$ with the following properties:

(T1) $\mathcal{M}_{\varepsilon, \delta}$ attracts $\widetilde{\mathcal{B}}_{\varepsilon}$ with an exponential rate which is uniform with respect to $\varepsilon$ and $\delta$, that is,

$$
\operatorname{dist}_{\mathcal{H}_{\varepsilon}^{0}}\left(S_{\varepsilon, \delta}(t) \widetilde{\mathcal{B}}_{\varepsilon}, \mathcal{M}_{\varepsilon, \delta}\right) \leq M_{1} e^{-\omega t}, \quad \forall t \geq 0
$$

for some $\omega>0$. 
(T2) The fractal dimension of $\mathcal{M}_{\varepsilon, \delta}$ is uniformly bounded with respect to $\varepsilon$ and $\delta$, that is,

$$
\operatorname{dim}_{\mathcal{H}_{\varepsilon}^{0}}\left[\mathcal{M}_{\varepsilon, \delta}\right] \leq M_{2} .
$$

(T3) The symmetric Hausdorff distance in $\mathcal{H}_{\varepsilon}^{0}$ between $\mathcal{M}_{\varepsilon, \delta}$ and $\mathcal{L}_{\varepsilon, \delta} \mathcal{M}_{0,0}$ satisfies

$$
\operatorname{dist}_{\mathcal{H}_{\varepsilon}^{0}}^{\mathrm{sym}}\left(\mathcal{M}_{\varepsilon, \delta}, \mathcal{L}_{\varepsilon, \delta} \mathcal{M}_{0,0}\right) \leq M_{3}(\varepsilon+\delta)^{\tau},
$$

for some $\tau \in\left(0, \frac{1}{2}\right]$.

The positive constants $\omega, \tau$ and $M_{j}$ are independent of $\varepsilon$ and $\delta$, and they can be explicitly calculated.

Remark. Owing to (H2) and the continuity of $S_{\varepsilon, \delta}(t)$, the inclusion $S_{\varepsilon, \delta}(t) \widetilde{\mathcal{B}}_{\varepsilon} \subset \widetilde{\mathcal{B}}_{\varepsilon}$ holds for all $t \geq t^{*}$. In fact, in most practical cases $\widetilde{\mathcal{B}}_{\varepsilon}=\mathcal{B}_{\varepsilon}$ (for instance, when both $X^{2}$ and $Y^{2}$ are reflexive).

Remark. In particular, hypotheses (H1)-(H3) entail that $\mathcal{M}_{\varepsilon, \delta}$ is a robust family of discrete exponential attractors for the maps $S_{\varepsilon, \delta}: \widetilde{\mathcal{B}}_{\varepsilon} \rightarrow \widetilde{\mathcal{B}}_{\varepsilon}$.

Remark. It is worth mentioning that, in concrete applications, hypotheses (H3) and (H4) are usually verified by the same token. Besides, with reference to (H2), it is often possible to decompose the map $S_{\varepsilon, \delta}$ into the sum $S_{\varepsilon, \delta}=\widehat{L}_{\varepsilon, \delta}+\widehat{K}_{\varepsilon, \delta}$, such that, for every $z_{1}, z_{2} \in \mathcal{B}_{\varepsilon}$,

$$
L_{\varepsilon, \delta}\left(z_{1}, z_{2}\right)=\widehat{L}_{\varepsilon, \delta} z_{1}-\widehat{L}_{\varepsilon, \delta} z_{2} \quad \text { and } \quad N_{\varepsilon, \delta}\left(z_{1}, z_{2}\right)=\widehat{N}_{\varepsilon, \delta} z_{1}-\widehat{N}_{\varepsilon, \delta} z_{2} .
$$

Before going to the proof, note that our Theorem applies as well to the simpler situation when $\varepsilon_{0}=0$, i.e., when the perturbation is not singular any longer. The map $\mathcal{J}_{\delta}$ can be taken to be the null map, and both $\mathcal{L}_{0, \delta}$ and $\Pi_{0}$ are the identity maps. In fact, in that case, the theorem is just a slight generalization of the abstract results contained in [4, 5, 10,

It will also be apparent from the proof that the result generalizes to the case when both $\varepsilon$ and $\delta$ are vectors. Clearly, one has to replace $\varepsilon$ and $\delta$ in the estimates with their euclidean norms.

\section{Proof of the Theorem}

Some passages appearing in the proof are borrowed from [5, 6. Nonetheless, for the sake of clarity, we have chosen to report all the details.

Up to rescaling $\varepsilon_{0}$ and $\delta_{0}$ (and, consequently, redefining accordingly the constants $\Lambda_{j}$ and $M_{j}$ ), we can always assume $\varepsilon_{0}+\delta_{0}<1$. Throughout the proof, let $C>0$ denote a generic constant independent of $\varepsilon$ and $\delta$.

The first task is to build a (discrete) exponential attractor $\mathcal{M}_{0,0}^{d}$ for the map $S_{0,0}$. To accomplish this purpose, we define by induction on $n \in \mathbb{N}$ a family of sets $V_{n} \subset S_{0,0}^{n} \mathcal{B}_{0}$. Fix an arbitrary $b_{0} \in \mathcal{B}_{0}$. Since $\mathcal{B}_{0}$ is bounded in $\mathcal{H}_{0}^{0}$, we choose $R>0$ such that $\mathbb{B}_{0}^{0}\left(b_{0}, R\right) \supset \mathcal{B}_{0}$, and we set $V_{0}=\left\{b_{0}\right\}$. Defining

$$
R_{n}=R\left(\frac{1}{2}+\kappa\right)^{n},
$$

we assume that $V_{n}$ is an $R_{n}$-net of $S_{0,0}^{n} \mathcal{B}_{0}$ in $\mathcal{H}_{0}^{0}$ such that card $\left[V_{n}\right] \leq N_{0}^{n}$, where

$$
N_{0}=\max \left\{3, \mathcal{N}_{\frac{1-2 \kappa}{4 \Lambda_{1}}}\left(\mathbb{B}_{\varepsilon_{0}}^{1}(0,1), \mathcal{H}_{\varepsilon_{0}}^{0}\right)\right\} .
$$


Note that the inequality

$$
\mathcal{N}_{r}\left(\mathbb{B}_{\varepsilon}^{1}(0,1), \mathcal{H}_{\varepsilon}^{0}\right) \leq \mathcal{N}_{r}\left(\mathbb{B}_{\varepsilon_{0}}^{1}(0,1), \mathcal{H}_{\varepsilon_{0}}^{0}\right)
$$

holds for every $r>0$ and every $\varepsilon \in\left[0, \varepsilon_{0}\right]$.

Then, we construct the $R_{n+1}$-net $V_{n+1}$ of $S_{0,0}^{n+1} \mathcal{B}_{0}$ in $\mathcal{H}_{0}^{0}$ in the following manner. For any fixed $b \in V_{n}$, assumption (H2) yields

$$
K_{0,0}\left(b, \mathbb{B}_{0}^{0}\left(b, R_{n}\right) \cap \mathcal{B}_{0}\right) \subset \mathbb{B}_{0}^{1}\left(0, \Lambda_{1} R_{n}\right) .
$$

Due to the compact embedding $\mathcal{H}_{0}^{1} \Subset \mathcal{H}_{0}^{0}$, let $W_{n}(b)$ be an $R_{n} \frac{1-2 \kappa}{4}$-net of $\mathbb{B}_{0}^{1}\left(0, \Lambda_{1} R_{n}\right)$ in $\mathcal{H}_{0}^{0}$ with the minimum number of balls. The cardinality of $W_{n}(b)$ is independent of $n$. Indeed,

$$
\begin{aligned}
\operatorname{card}\left[W_{n}(b)\right] & \leq \mathcal{N}_{R_{n} \frac{1-2 \kappa}{4}}\left(\mathbb{B}_{0}^{1}\left(0, \Lambda_{1} R_{n}\right), \mathcal{H}_{0}^{0}\right) \\
& \leq \mathcal{N}_{\frac{1-2 \kappa}{4 \Lambda_{1}}}\left(\mathbb{B}_{0}^{1}(0,1), \mathcal{H}_{0}^{0}\right) \\
& \leq N_{0}
\end{aligned}
$$

Note that $S_{0,0} b-W_{n}(b)$ is an $\frac{R_{n+1}}{2}$-net of $S_{0,0}\left(\mathbb{B}_{0}^{0}\left(b, R_{n}\right) \cap \mathcal{B}_{0}\right)$, for, given $x \in$ $\mathbb{B}_{0}^{0}\left(b, R_{n}\right) \cap \mathcal{B}_{0}$, we can find $b_{j} \in W_{n}(b)$ such that

$$
\left\|K_{0,0}(b, x)-b_{j}\right\|_{\mathcal{H}_{0}^{0}} \leq R_{n} \frac{1-2 \kappa}{4} .
$$

Hence, exploiting the decomposition (H2),

$$
\begin{aligned}
\left\|S_{0,0} x-S_{0,0} b+b_{j}\right\|_{\mathcal{H}_{0}^{0}} & =\left\|L_{0,0}(b, x)+K_{0,0}(b, x)-b_{j}\right\|_{\mathcal{H}_{0}^{0}} \\
& \leq \kappa\|x-b\|_{\mathcal{H}_{0}^{0}}+R_{n} \frac{1-2 \kappa}{4} \leq \frac{R_{n+1}}{2} .
\end{aligned}
$$

Thus, $\bigcup_{b \in V_{n}}\left(S_{0,0} b-W_{n}(b)\right)$ is an $\frac{R_{n+1}}{2}$-net of $S_{0,0}^{n+1} \mathcal{B}_{0}$, with

$$
\operatorname{card}\left[\bigcup_{b \in V_{n}}\left(S_{0,0} b-W_{n}(b)\right)\right] \leq N_{0} \operatorname{card}\left[V_{n}\right] \leq N_{0}^{n+1} .
$$

Doubling the radius, that is, taking balls of radius $R_{n+1}$, we get a covering of $S_{0,0}^{n+1} \mathcal{B}_{0}$ whose balls are centered in $S_{0,0}^{n+1} \mathcal{B}_{0}$. The corresponding net is $V_{n+1}$. Summing up, we have constructed a family of sets $V_{n} \subset S_{0,0}^{n} \mathcal{B}_{0}$ such that

$$
\operatorname{card}\left[V_{n}\right] \leq N_{0}^{n} \quad \text { and } \quad \operatorname{dist}_{\mathcal{H}_{0}^{0}}\left(S_{0,0}^{n} \mathcal{B}_{0}, V_{n}\right) \leq R_{n} .
$$

By a further inductive procedure, we build a second family of sets

$$
E_{0}=V_{0}, \quad E_{n+1}=S_{0,0} E_{n} \cup V_{n+1} .
$$

Clearly, $E_{n}$ is an $R_{n}$-net of $S_{0,0}^{n} \mathcal{B}_{0}$. We can easily check the following properties:

$$
\begin{aligned}
& E_{n} \subset S_{0,0}^{n} \mathcal{B}_{0}, \quad S_{0,0} E_{n} \subset E_{n+1}, \\
& \operatorname{card}\left[E_{n}\right] \leq N_{0}^{n+1}, \\
& \operatorname{dist}_{\mathcal{H}_{0}^{0}}\left(S_{0,0}^{n} \mathcal{B}_{0}, E_{n}\right) \leq R_{n} .
\end{aligned}
$$

Thus, setting

$$
\mathcal{M}_{0,0}^{\prime d}=\bigcup_{n \in \mathbb{N}} E_{n}, \quad \mathcal{M}_{0,0}^{d}=\overline{\mathcal{M}_{0,0}^{\prime d}} \mathcal{H}_{0}^{0},
$$

property (11) together with (H2) yield $S_{0,0} \mathcal{M}_{0,0}^{d} \subset \mathcal{M}_{0,0}^{d} \subset \widetilde{\mathcal{B}}_{0}$. Moreover, (3) entails

$$
\operatorname{dist}_{\mathcal{H}_{0}^{0}}\left(S_{0,0}^{n} \widetilde{\mathcal{B}}_{0}, \mathcal{M}_{0,0}^{d}\right) \leq R_{n}
$$


Finally, fix $0<r \ll 1$. Then there exists $n \in \mathbb{N}$ such that $R_{n} \geq r$, and $R_{n+1}<r$. It is apparent that $n \rightarrow \infty$ as $r \rightarrow 0$. From the definition of $\mathcal{M}_{0,0}^{\prime d}$, we deduce that

$$
\mathcal{N}_{r}\left(\mathcal{M}_{0,0}^{\prime d}, \mathcal{H}_{0}^{0}\right) \leq \mathcal{N}_{r}\left(\bigcup_{k \leq n} E_{k}, \mathcal{H}_{0}^{0}\right)+\mathcal{N}_{r}\left(\bigcup_{k>n} E_{k}, \mathcal{H}_{0}^{0}\right)
$$

Observe that $\bigcup_{k>n} E_{k} \subset S_{0,0}^{n+1} \mathcal{B}_{0}$ and $V_{n+1}$ is an $R_{n+1}$-net of $S_{0,0}^{n+1} \mathcal{B}_{0}$. Hence, (2) yields

$$
\mathcal{N}_{r}\left(\mathcal{M}_{0,0}^{\prime d}, \mathcal{H}_{0}^{0}\right) \leq \sum_{k=0}^{n} \operatorname{card}\left[E_{k}\right]+\operatorname{card}\left[V_{n+1}\right] \leq N_{0}^{n+2}
$$

and it is immediate to check that

$$
\frac{\ln \mathcal{N}_{r}\left(\mathcal{M}_{0,0}^{\prime d}, \mathcal{H}_{0}^{0}\right)}{\ln \frac{1}{r}} \leq \frac{(n+2) \ln N_{0}}{\ln \frac{1}{R}+n \ln \left(\frac{2}{2 \kappa+1}\right)} .
$$

Thus, taking the limit $r \rightarrow 0$, we find the inequality

$$
\operatorname{dim}_{\mathcal{H}_{0}^{0}}\left[\mathcal{M}_{0,0}^{\prime d}\right] \leq \frac{\ln N_{0}}{\ln \left(\frac{2}{2 \kappa+1}\right)},
$$

and we conclude that $\mathcal{M}_{0,0}^{d}$ (which is clearly compact) is an exponential attractor for $S_{0,0}$ on $\widetilde{\mathcal{B}}_{0}$.

The next step is to construct $\mathcal{M}_{\varepsilon, \delta}^{d}$ from $\mathcal{M}_{0,0}^{d}$. Here, we exploit (1) to obtain a family $\widehat{E}_{n}$ from $E_{n}$ with the following properties:

$$
\widehat{E}_{n} \subset \mathcal{B}_{0} \quad \text { such that } \quad S_{0,0}^{n} \widehat{E}_{n}=E_{n}, \quad \operatorname{card}\left[\widehat{E}_{n}\right] \leq N_{0}^{n+1} .
$$

By the very definition of $\Pi_{\varepsilon}$, there exists $\widehat{E}_{n}(\varepsilon) \subset \mathcal{B}_{\varepsilon}$ such that

$$
\Pi_{\varepsilon} \widehat{E}_{n}(\varepsilon)=\widehat{E}_{n}, \quad \operatorname{card}\left[\widehat{E}_{n}(\varepsilon)\right] \leq N_{0}^{n+1} .
$$

Then we introduce the two subsets of $\mathcal{H}_{\varepsilon}^{0}$,

$$
\widetilde{E}_{n}(\varepsilon, \delta)=S_{\varepsilon, \delta}^{n} \widehat{E}_{n}(\varepsilon) \quad \text { and } \quad \widehat{E}_{n}(\varepsilon, \delta)=\mathcal{L}_{\varepsilon, \delta} E_{n} .
$$

Setting $\nu=\min \left\{\beta, \frac{1}{2}\right\}$, we shall prove the estimate

$$
\operatorname{dist}_{\mathcal{H}_{\varepsilon}^{0}}\left(S_{\varepsilon, \delta}^{n} \mathcal{B}_{\varepsilon}, \widetilde{E}_{n}(\varepsilon, \delta)\right) \leq C \Lambda_{2}^{n}(\varepsilon+\delta)^{\nu}+C\left(\frac{1}{2}+\kappa\right)^{\alpha n}, \quad \forall n \in \mathbb{N} .
$$

Fix $z \in \mathcal{B}_{\varepsilon}$. Thanks to (H3), and treating apart the case $n=0$, we easily see that

$$
\left\|S_{\varepsilon, \delta}^{n} z-\mathcal{L}_{\varepsilon, \delta} S_{0,0}^{n} \Pi_{\varepsilon} z\right\|_{\mathcal{H}_{\varepsilon}^{0}} \leq C \Lambda_{2}^{n}(\varepsilon+\delta)^{\nu},
$$

for some $C \geq 1$. Since $\mathcal{J}_{\delta}$ is Hölder continuous of exponent $\alpha$ from $\mathcal{B}_{0}$ into $Y^{0}$, it follows that $\mathcal{L}_{\varepsilon, \delta} E_{n}$ is a $C\left(\frac{1}{2}+\kappa\right)^{\alpha n}$-net of $\mathcal{L}_{\varepsilon, \delta} S_{0,0}^{n} \mathcal{B}_{0}$ in $\mathcal{H}_{\varepsilon}^{0}$. Thus, there exists $\bar{z} \in E_{n}$ such that

$$
\left\|\mathcal{L}_{\varepsilon, \delta} S_{0,0}^{n} \Pi_{\varepsilon} z-\mathcal{L}_{\varepsilon, \delta} \bar{z}\right\|_{\mathcal{H}_{\varepsilon}^{0}} \leq C\left(\frac{1}{2}+\kappa\right)^{\alpha n} .
$$


By the definition of $\widehat{E}_{n}(\varepsilon)$, we can find $\hat{z} \in \widehat{E}_{n}(\varepsilon)$ such that $\bar{z}=S_{0,0}^{n} \Pi_{\varepsilon} \hat{z}$. Reasoning as before, we have

$$
\left\|S_{\varepsilon, \delta}^{n} \hat{z}-\mathcal{L}_{\varepsilon, \delta} \bar{z}\right\|_{\mathcal{H}_{\varepsilon}^{0}}=\left\|S_{\varepsilon, \delta}^{n} \hat{z}-\mathcal{L}_{\varepsilon, \delta} S_{0,0}^{n} \Pi_{\varepsilon} \hat{z}\right\|_{\mathcal{H}_{\varepsilon}^{0}} \leq C \Lambda_{2}^{n}(\varepsilon+\delta)^{\nu} .
$$

Collecting the three above inequalities, we deduce (6), as claimed.

Without loss of generality, we now assume $\Lambda_{2} \geq 1$. Then, since $\varepsilon_{0}+\delta_{0}<1$, setting

$$
n_{0}=n_{0}(\varepsilon, \delta)=\frac{\nu \ln (\varepsilon+\delta)}{\alpha \ln \left(\frac{1}{2}+\kappa\right)-\ln \Lambda_{2}}>0
$$

and

$$
\sigma=\frac{\nu \alpha \ln \left(\frac{1}{2}+\kappa\right)}{\alpha \ln \left(\frac{1}{2}+\kappa\right)-\ln \Lambda_{2}} \in\left(0, \frac{1}{2}\right]
$$

we find the equality

$$
\Lambda_{2}^{n_{0}}(\varepsilon+\delta)^{\nu}=\left(\frac{1}{2}+\kappa\right)^{\alpha n_{0}}=(\varepsilon+\delta)^{\sigma} .
$$

We now distinguish two cases. When $n \leq n_{0}(\varepsilon, \delta)$, we set $E_{n}(\varepsilon, \delta)=\widetilde{E}_{n}(\varepsilon, \delta)$. Thanks to (H3), (6) and the choice of $n_{0}(\varepsilon, \delta)$ and $\sigma$, there hold

$$
\operatorname{dist}_{\mathcal{H}_{\varepsilon}^{0}}^{\text {sym }}\left(E_{n}(\varepsilon, \delta), \mathcal{L}_{\varepsilon, \delta} E_{n}\right) \leq C(\varepsilon+\delta)^{\sigma}, \quad \forall n \leq n_{0}(\varepsilon, \delta),
$$

and

$$
\operatorname{dist}_{\mathcal{H}_{\varepsilon}^{0}}\left(S_{\varepsilon, \delta}^{n} \mathcal{B}_{\varepsilon}, E_{n}(\varepsilon, \delta)\right) \leq C\left(\frac{1}{2}+\kappa\right)^{\alpha n}, \quad \forall n \leq n_{0}(\varepsilon, \delta)
$$

For the case $n>n_{0}(\varepsilon, \delta)$, the sets $E_{n}(\varepsilon, \delta)$ are constructed by induction, paralleling the construction of $E_{n}$, starting from the initial step $E_{\left[n_{0}\right]}(\varepsilon, \delta)$ (in particular, we ask (8) to hold for all $n$ ). Then, for every $n \in \mathbb{N}$, the family $E_{n}(\varepsilon, \delta)$ turns out to fulfill

$$
E_{n}(\varepsilon, \delta) \subset S_{\varepsilon, \delta}^{n} \mathcal{B}_{\varepsilon}, \quad S_{\varepsilon, \delta} E_{n}(\varepsilon, \delta) \subset E_{n+1}(\varepsilon, \delta), \quad \operatorname{card}\left[E_{n}(\varepsilon, \delta)\right] \leq N_{0}^{n+2},
$$

and

$$
\operatorname{dist}_{\mathcal{H}_{\varepsilon}^{0}}\left(S_{\varepsilon, \delta}^{n} \mathcal{B}_{\varepsilon}, E_{n}(\varepsilon, \delta)\right) \leq C\left(\frac{1}{2}+\kappa\right)^{\alpha n} .
$$

At this point, we define

$$
\mathcal{M}_{\varepsilon, \delta}^{\prime d}=\bigcup_{n \in \mathbb{N}} E_{n}(\varepsilon, \delta), \quad \mathcal{M}_{\varepsilon, \delta}^{d}={\overline{\mathcal{M}_{\varepsilon, \delta}^{\prime d}}}_{\mathcal{H}_{\varepsilon}^{0}}
$$

Thanks to (9) and (H2), it is apparent that $S_{\varepsilon, \delta} \mathcal{M}_{\varepsilon, \delta}^{d} \subset \mathcal{M}_{\varepsilon, \delta}^{d} \subset \widetilde{\mathcal{B}}_{\varepsilon}$, and, by (10),

$$
\operatorname{dist}_{\mathcal{H}_{\varepsilon}^{0}}\left(S_{\varepsilon, \delta}^{n} \widetilde{\mathcal{B}}_{\varepsilon}, \mathcal{M}_{\varepsilon, \delta}^{d}\right) \leq C\left(\frac{1}{2}+\kappa\right)^{\alpha n} .
$$

The bound on the fractal dimension

$$
\operatorname{dim}_{\mathcal{H}_{\varepsilon}^{0}}\left[\mathcal{M}_{\varepsilon, \delta}^{d}\right] \leq C
$$

is a straightforward consequence of (9). To obtain the uniform estimate

$$
\operatorname{dist}_{\mathcal{H}_{\varepsilon}^{0}}^{\mathrm{sym}}\left(\mathcal{M}_{\varepsilon, \delta}^{d}, \mathcal{L}_{\varepsilon, \delta} \mathcal{M}_{0,0}^{d}\right) \leq C(\varepsilon+\delta)^{\sigma},
$$


it suffices to prove

$$
\operatorname{dist}_{\mathcal{H}_{\varepsilon}^{0}}\left(\mathcal{M}_{\varepsilon, \delta}^{\prime d}, \mathcal{L}_{\varepsilon, \delta} \mathcal{M}_{0,0}^{d}\right) \leq C(\varepsilon+\delta)^{\sigma},
$$

since the other inequality follows in the same manner, and (13) is then a consequence of the definition of $\mathcal{M}_{\varepsilon, \delta}^{d}$. Hence, fix $z \in \mathcal{M}_{\varepsilon, \delta}^{\prime d}$. By definition, there exists $n \in \mathbb{N}$ such that $z \in E_{n}(\varepsilon, \delta)$. If $n \leq n_{0}(\varepsilon, \delta)$, the claim immediately follows from (7). In the other case, due to $(\mathrm{H} 2), z \in S_{\varepsilon, \delta}^{\left[n_{0}\right]} \mathcal{B}_{\varepsilon}$, and we find $\bar{z} \in \mathcal{B}_{\varepsilon}$ such that $z=S_{\varepsilon, \delta}^{\left[n_{0}\right]} \bar{z}$. Therefore, from (H3) we get

$$
\left\|z-\mathcal{L}_{\varepsilon, \delta} S_{0,0}^{\left[n_{0}\right]} \Pi_{\varepsilon} \bar{z}\right\|_{\mathcal{H}_{\varepsilon}^{0}} \leq C \Lambda_{2}^{\left[n_{0}\right]}(\varepsilon+\delta)^{\sigma},
$$

where $C \geq 1$ appears to handle the possible case $\left[n_{0}\right]=0$. Moreover, in view of (44), the Hölder continuity of $\mathcal{J}_{\delta}$ and the choice of $\sigma$,

$$
\operatorname{dist}_{\mathcal{H}_{\varepsilon}^{0}}\left(\mathcal{L}_{\varepsilon, \delta} S_{0,0}^{\left[n_{0}\right]} \Pi_{\varepsilon} \bar{z}, \mathcal{L}_{\varepsilon, \delta} \mathcal{M}_{0,0}^{d}\right) \leq C(\varepsilon+\delta)^{\sigma} .
$$

Collecting these two inequalities, we reach the desired conclusion.

So far, we have found a family of exponential attractors $\mathcal{M}_{\varepsilon, \delta}^{d}$ for $S_{\varepsilon, \delta}$ in $\widetilde{\mathcal{B}}_{\varepsilon}$ that, in addition, satisfies (11)-13).

We are left to make the passage from the discrete to the continuous case. To this aim, define

$$
\mathcal{M}_{\varepsilon, \delta}=\bigcup_{t \in\left[t^{*}, 2 t^{*}\right]} S_{\varepsilon, \delta}(t) \mathcal{M}_{\varepsilon, \delta}^{d} .
$$

Thanks to (H6), it is apparent that $\mathcal{M}_{\varepsilon, \delta}$ is compact. Moreover, due to (H2) and the corresponding properties for the discrete exponential attractors, $S_{\varepsilon, \delta}(t) \mathcal{M}_{\varepsilon, \delta} \subset$ $\mathcal{M}_{\varepsilon, \delta} \subset \widetilde{\mathcal{B}}_{\varepsilon}$, for any $t \geq 0$. The exponential attraction property (T1) is obtained by (44), (11) and the uniform Lipschitz continuity of $S_{\varepsilon, \delta}$ at any fixed time with respect to the initial data provided by (H5), whereas the control on the fractal dimension (T2) easily follows from (5), (12) and assumption (H6). Indeed,

$$
\operatorname{dim}_{\mathcal{H}_{\varepsilon}^{0}}\left[\mathcal{M}_{\varepsilon, \delta}\right] \leq \frac{1}{\vartheta} \operatorname{dim}_{\mathcal{H}_{\varepsilon}^{0}}\left[\left[t^{*}, 2 t^{*}\right] \times \mathcal{M}_{\varepsilon, \delta}^{d}\right] \leq \frac{1}{\vartheta}\left(1+\operatorname{dim}_{\mathcal{H}_{\varepsilon}^{0}}\left[\mathcal{M}_{\varepsilon, \delta}^{d}\right]\right) \leq C .
$$

Finally, concerning the robustness of the family (T3), we exploit (H1), (H4) and (H5), thereby getting

$$
\left\|S_{\varepsilon, \delta}(t) z-\mathcal{L}_{\varepsilon, \delta} S_{0,0}(t) \bar{z}\right\|_{\mathcal{H}_{\varepsilon}^{0}} \leq \Lambda_{3}(\varepsilon+\delta)^{\gamma}+C\left\|\Pi_{\varepsilon} z-\bar{z}\right\|_{\mathcal{H}_{0}^{0}}^{\alpha}, \quad \forall t \in\left[t^{*}, 2 t^{*}\right],
$$

for any $z \in \mathcal{M}_{\varepsilon, \delta}^{d}$ and $\bar{z} \in \mathcal{M}_{0,0}^{d}$. Then, in view of (13), we obtain

$$
\begin{aligned}
\operatorname{dist}_{\mathcal{H}_{\varepsilon}^{0}}\left(\mathcal{M}_{\varepsilon, \delta}, \mathcal{L}_{\varepsilon, \delta} \mathcal{M}_{0,0}\right) & \leq \sup _{t \in\left[t^{*}, 2 t^{*}\right]} \sup _{z \in \mathcal{M}_{\varepsilon, \delta}^{d}} \inf _{s \in\left[t^{*}, 2 t^{*}\right]} \inf _{\bar{z} \in \mathcal{M}_{0,0}^{d}}\left\|S_{\varepsilon, \delta}(t) z-\mathcal{L}_{\varepsilon, \delta} S_{0,0}(s) \bar{z}\right\|_{\mathcal{H}_{\varepsilon}^{0}} \\
& \leq \sup _{t \in\left[t^{*}, 2 t^{*}\right]} \sup _{z \in \mathcal{M}_{\varepsilon, \delta}^{d}} \inf _{\bar{z} \in \mathcal{M}_{0,0}^{d}}\left\|S_{\varepsilon, \delta}(t) z-\mathcal{L}_{\varepsilon, \delta} S_{0,0}(t) \bar{z}\right\|_{\mathcal{H}_{\varepsilon}^{0}} \\
& \leq M_{3}(\varepsilon+\delta)^{\tau},
\end{aligned}
$$

where $\tau=\min \{\gamma, \alpha \sigma\}$.

\section{Some Applications}

We finally report some possible applications of the theorem to concrete examples arising from PDE. We will just focus on some already-known instances, giving some hints of how to treat the problems in this new setting. As we mentioned in the 
Introduction, this technique allows us to recover all known results with less effort. Other models will be investigated within this approach in forthcoming papers.

In the sequel, let $\Omega \subset \mathbb{R}^{3}$ be a smooth domain and let $A=-\Delta$ be the Laplace operator on $L^{2}(\Omega)$ with domain $\mathcal{D}(A)=H^{2}(\Omega) \cap H_{0}^{1}(\Omega)$. Then, for $s \in \mathbb{R}$, we introduce the Hilbert spaces $H_{s}=\mathcal{D}\left(A^{s / 2}\right)$.

4.1. Weakly damped wave equations. For $\varepsilon \geq 0$, consider the equation

$$
\left\{\begin{array}{l}
\varepsilon u_{t t}+u_{t}+A u+\phi(u)=0, \\
u(0)=u_{0}, \\
\varepsilon u_{t}(0)=\varepsilon u_{1}
\end{array}\right.
$$

in $\Omega \times \mathbb{R}^{+}$. Here, $\phi$ is a smooth function with cubic controlled growth, satisfying some further dissipation conditions. This equation defines a strongly continuous semigroup $S_{\varepsilon}(t)$ on the phase-space $H_{1} \times H_{0}$ (or $H_{1}$ when $\varepsilon=0$ ). In order to find a robust family of exponential attractors for this problem, the authors introduce in [6] the spaces

$$
\mathcal{V}_{\varepsilon}= \begin{cases}H_{1} \times H_{0}, & \text { if } \quad \varepsilon>0 \\ H_{1} \times H_{-1}, & \text { if } \quad \varepsilon=0\end{cases}
$$

endowed with the norms

$$
\|(u, v)\|_{\mathcal{V}_{\varepsilon}}^{2}=\|u\|_{H_{1}}^{2}+\varepsilon\|v\|_{H_{0}}^{2}+\|v\|_{H_{-1}}^{2} .
$$

Next, they consider the infinite-dimensional submanifold $\mathcal{E}_{0}$ of $\mathcal{V}_{0}$ given by

$$
\mathcal{E}_{0}=\left\{(u, v): u \in H_{1}, v=-A u-\phi(u)\right\} .
$$

Then setting

$$
\widetilde{\mathcal{V}}_{\varepsilon}= \begin{cases}\mathcal{V}_{\varepsilon}, & \text { if } \quad \varepsilon>0, \\ \mathcal{E}_{0}, & \text { if } \quad \varepsilon=0,\end{cases}
$$

they construct the semigroups $\Sigma_{\varepsilon}(t)$ on $\widetilde{\mathcal{V}}_{\varepsilon}$ as follows:

$$
\Sigma_{\varepsilon}(t)(u, v)= \begin{cases}S_{\varepsilon}(t)(u, v), & \text { if } \quad \varepsilon>0 \\ \left(S_{0}(t) u,-A S_{0}(t) u-\phi\left(S_{0}(t) u\right)\right), & \text { if } \quad \varepsilon=0 .\end{cases}
$$

Finally, starting from a more regular subset $\mathcal{C}_{\varepsilon}$ of $\widetilde{\mathcal{V}}_{\varepsilon}$, they show that there exists a family of exponential attractors for these new semigroups $\Sigma_{\varepsilon}(t)$ on $\mathcal{C}_{\varepsilon}$, which is robust with respect to the limit $\varepsilon \rightarrow 0$.

A similar result holds for a singular nonlinearity $\phi \in C^{3}(-1,1)$ satisfying $\phi(0)=$ $0, \lim _{x \rightarrow \pm 1} \phi(x)= \pm \infty$, and $\lim _{x \rightarrow \pm 1} \phi^{\prime}(x)=+\infty$ (see [8]).

In our setting, the result can be recovered putting

$$
X^{0}=H_{1}, \quad X^{1}=H_{2}, \quad X^{2}=H_{3}, \quad Y^{0}=H_{0}, \quad Y^{1}=H_{1}, \quad Y^{2}=H_{2} .
$$

The map $\mathcal{J}_{0}\left(\delta_{0}=0\right.$ in that case) is given by

$$
\mathcal{J}_{0}(u)=-A u-\phi(u) .
$$

Then (H1) $-(\mathrm{H} 6)$ are verified, with $\alpha=\beta=\gamma=\frac{1}{2}$ and $\vartheta=1$.

It should be noted that the idea of [6, 8, loosely speaking, is to "lift" the whole limiting problem. Conversely, in our approach, we work in the natural spaces, and we just lift the exponential attractor of the limiting equation. 
4.2. Cahn-Hilliard type equations. The following hyperbolic relaxation of the viscous Cahn-Hilliard equation in $\Omega \times \mathbb{R}^{+}$has been considered in [7]:

$$
\left\{\begin{array}{l}
\varepsilon u_{t t}+u_{t}+A\left(A u+\phi(u)+\delta u_{t}\right)=0 \\
u(0)=u_{0} \\
\varepsilon u_{t}(0)=\varepsilon u_{1}
\end{array}\right.
$$

Here, $\varepsilon \geq 0, \delta \geq \varepsilon$, and $\phi$ is as in Section 4.1. This equation is shown to generate a strongly continuous semigroup on the phase-space $H_{2} \times H_{0}$ (or $H_{2}$ when $\varepsilon=0$ ). Again, there exists a family of exponential attractors which is robust with respect to the limit $\delta \rightarrow 0$ (which forces $\varepsilon \rightarrow 0$ ). The procedure to get the result is similar to the one used in Section 4.1, although more complicated, due to the presence of two parameters. We address the reader to [7] for more details.

With our method, we find the same result by setting

$$
X^{0}=H_{2}, \quad X^{1}=H_{3}, \quad X^{2}=H_{6}, \quad Y^{0}=H_{0}, \quad Y^{1}=H_{1}, \quad Y^{2}=H_{4} .
$$

The map $\mathcal{J}_{\delta}$ is given by

$$
\mathcal{J}_{\delta}(u)=-(\mathbb{I}+\delta A)^{-1} A(A u+\phi(u)) .
$$

Then (H1)-(H6) are verified, with $\alpha=\beta=\gamma=\frac{1}{2}$ and $\vartheta=1$.

It is worth noting that the map $\mathcal{J}_{\delta}$ is dictated by the equation, as well as the map $\mathcal{J}_{0}$ of the previous example. So, the only arbitrariness is the correct choice of the spaces $X^{1}, X^{2}, Y^{1}$ and $Y^{2}$. However, this is suggested by the requirement that (H1) and (H2) hold.

\section{REFERENCES}

[1] L. Dung, B. Nicolaenko, Exponential attractors in Banach spaces, J. Dynam. Differential Equations 13 (2001), 791-806. MR1860286 (2002h:37158)

[2] A. Eden, C. Foias, B. Nicolaenko, R. Temam, Exponential attractors for dissipative evolution equations, Masson, Paris, 1994. MR.1335230 (96i:34148)

[3] M. Efendiev, A. Miranville, S. Zelik, Exponential attractors for a nonlinear reaction-diffusion system in $\mathbb{R}^{3}$, C.R. Acad. Sci. Paris Sér. I Math. 330 (2000), 713-718. MR.1763916 (2001c:35039)

[4] M. Efendiev, A. Miranville, S. Zelik, Exponential attractors for a singularly perturbed CahnHilliard system, Math. Nachr. 272 (2004), 11-31. MR2079758 (2005h:37195)

[5] M. Efendiev, A. Miranville, S. Zelik, Global and exponential attractors for nonlinear reactiondiffusion systems in unbounded domains, Proc. Roy. Soc. Edinburgh Sect. A 134, (2004), 271-315. MR2056285 (2005h:37196)

[6] P. Fabrie, C. Galusinski, A. Miranville, S. Zelik, Uniform exponential attractors for a singularly perturbed damped wave equation, Discrete Contin. Dynam. Systems 10 (2004), 211-238. MR2026192

[7] S. Gatti, M. Grasselli, A. Miranville, V. Pata, Hyperbolic relaxation of the viscous CahnHilliard equation in 3-D, Math. Models Methods Appl. Sci., 15 (2005), 165-198. MR2119676

[8] M. Grasselli, A. Miranville, V. Pata, S. Zelik, Well-posedness and long time behavior of a parabolic-hyperbolic phase-field system with singular potentials. To appear in Mathematische Nachrichten.

[9] L.A. Lyusternik, M.I. Vishik, Regular degeneration and boundary layer for linear differential equations with small parameter, Trans. Amer. Math. Soc. 20 (1962), 239-364. 
[10] A. Miranville, S. Zelik, Robust exponential attractors for Cahn-Hilliard type equations with singular potentials, Math. Methods Appl. Sci. 27 (2004), 545-582. MR2041814 (2005b:37191)

[11] R. Temam, Infinite-dimensional dynamical systems in mechanics and physics, SpringerVerlag, New York, 1988. MR0953967 (89m:58056)

Dipartimento di Matematica, Università di Ferrara, Via Machiavelli 35, I-44100 FerRARA, ITALY

E-mail address: s.gatti@economia.unife.it

Dipartimento di Matematica "F.Brioschi", Politecnico di Milano, Via Bonardi 9, I20133 Milano, Italy

E-mail address: maugra@mate.polimi.it

Université de Poitiers, Laboratoire de Mathématiques et Applications, UMR CNRS 6086 - SP2Mi, Boulevard Marie et Pierre Curie - Téléport 2, F-86962 Chasseneuil Futuroscope Cedex, France

E-mail address: miranv@math.univ-poitiers.fr

Dipartimento di Matematica "F.Brioschi", Politecnico di Milano, Via Bonardi 9, I20133 Milano, Italy

E-mail address: pata@mate.polimi.it 